\title{
Study on the Important Role of Piano Accompaniment in the Performance of Ethnic Instruments
}

\author{
Zhenzhong Li \\ Xi’an Conservatory of Music, Shanxi Xi’an, 710061
}

Keywords: piano accompaniment; ethnic instrumental; performance skills; art guidance

\begin{abstract}
Ethnic musical instruments are the unique culture of the Chinese nation and the representative of Chinese culture. Therefore, the performance of ethnic musical instruments is also loved by more and more people. The accompaniment plays an important role in both vocal singing and instrumental performance; and the popularity of the piano makes the piano accompaniment become the first choice of daily singing, playing accompaniment. As a unique kind of musical instrument in China, the history of western musical instrument accompaniment is not long, but the piano accompaniment plays an important role in promoting the artistic and emotional perfection of Chinese instrumental music. This paper will study the important role of piano accompaniment in the performance of folk musical instruments.
\end{abstract}

The piano accompaniment, with its rich expression and harmony with instruments and human voices, is admired by vocal singing and instrumental performance. Although there is no orchestra majestic momentum and rich timbre in the piano accompaniment, but its convenient use, and with singing, players communicate directly, is the most direct advantage, and piano rich harmony texture, wide range, variety of timbre, etc, for the processing of detail, the expression of emotion can be more accurate in place, the directness of communication with singing and performers is the most direct advantage. Besides, the features like rich harmonic texture, wide range and variable timbre and so on make the handling of the details of the work, the expression of emotions can be more precise and accurate.

\section{Problems of piano accompaniment for ethnic instrumental music}

Due to the rapid development of the art examination in recent years, the study of ethnic instrumental music has emerged a trend of exam-oriented education, and the study of ethnic instrumental performance has been significantly enhanced, rather than the real interests in the study of ethnic instrumental music. As a result, the study of ethnic instrumental music is passive and mechanical, and cannot feel the charm of ethnic instrumental music deeply; moreover, many ethnic instrumental music teachers are lack of responsibility and formal professional guidance in the teaching process, and only provide some guidance for students' exam skills, but lack of education for their artistic accomplishment and quality, which seriously hinders students' future development.

\section{Artistic features of piano accompaniment for ethnic instrumental music}

As a kind of expressive features of western instruments, piano have the characteristics of western techniques on accompaniment orchestration, but for the accompaniment of ethnic instrumental music, it is also necessary to preserve the national characteristics of the accompaniment, analyze from music materials such as the melody, tonality and melody of the music, and use the thinking of multi-part music to achieve harmony and unity between the accompaniment and the solo part of ethnic instrumental music. Guzheng, for example, notably the present paper arrives at an inter pretation ", erhu violin "ballade of the north" and so on, the success of ethnic Musical Instruments and piano polyphonic music perfect match, in terms of tone, rhythm, speed, harmony, the national music style to the full performance of the national instrumental solo atmosphere foil, can not replace the role of mood. For example, famous guzheng music, night at maple bridge, famous erhu 
music, ballade of northern henan, etc, make a perfect match between national instruments and piano accompaniment on music in terms of timbre, rhythm, speed, harmony,and so on, the style of national music is fully displayed, and the effect on the atmosphere and emotional rendering of national instrumental solo is irreplaceable.

\section{The performance skills required for the piano accompaniment of ethnic instrumental music}

Shift tone, scale, arpeggios, chords, octaves, skilled shift tone, reasonable use of pedals and other piano playing skills, and also the piano accompaniment needs to have the basis of playing, if want to play the piano accompaniment of ethnic instrumental music well, it is also necessary to have an understanding of the range, timbre and performance characteristics of various ethnic instruments (such as erhu, guzheng, pipa, flute, ruan, etc.), reasonably control the accompaniment skills, volume and timbre, so as to neither beat the crowd, nor weaken the role of accompaniment. The advantage of the piano accompaniment lies in its strong artistic expression. After the accompaniment fully understands the musical instrument and musical structure of accompaniment, he can provide the music with an atmosphere of appropriate position, so as to mobilize the emotion of the main player and depict the due artistic conception of the music.

The introduction, interplay and tail parts of the national music have many scattered boards with long length and free rhythm, it needs skilful arpeggios, triplet, seven liaison playing skills to assist the piano accompanist to express their understanding of the music, so that the piano accompaniment can really play a better auxiliary role in the music, and through different ways of touching the keys, show the sound and sound effects in line with the national instruments, so that the two more harmonious integration. For example, the string sweeping technique of guzheng can be demonstrated by scraping, and the clear tone of the flute can be imitated by tremolo.

For the passages with strong conflicts in national music, it is necessary for the piano accompaniment to promote the atmosphere and timbre, it is necessary for the intensive accompaniment sound pattern, and the gradually strengthened tightening effect to highlight the long melody features of the main melody, making the contrast more intense and pushing the music to the climax step by step.

\section{The piano accompaniment and the national musical instrument master play needs the strong cooperation consciousness}

Cooperation -- it is the link between accompaniment and the master. First of all, we should jointly study the music style, harmonic texture and form structure of the music. From the complete impression to the detailed rhythm and intonation, we should have a relatively consistent understanding of the music and strengthen it sentence by sentence. From the separation of parts, to the combination, to increase mutual understanding, to overcome the difficulties and difficulties, from unfamiliarity to proficiency, from proficiency to ease, and finally to better and perfect expression of works.

\section{The piano accompaniment plays an important role in the performance of ethnic instrumental music}

\subsection{Piano accompaniment plays an important role in the teaching of ethnic instrumental music}

Teaching and learning is an important link in national instrumental music playing skills, as a competent national instrumental music teacher, mastering the piano accompaniment and ethnic instruments teaching skills are equally important, it is to gradually train students' artistic accomplishment and improve their basic teaching ability in class. Through the coordination and coordination of piano accompaniment and instrumental performance, students can understand the works more profoundly and finally bring the audience more profound auditory enjoyment.

The strict piano accompaniment should be rigorous and standard. The rhythm, speed, timbre and 
other music elements of the accompaniment should be based on the ethnic instrumental music. As a national instrumental teacher, in the course of music teaching, students should be taught to analyze the work and analyze the accompaniment, and the work should be handled from the point of music feeling, so that the musical instrument and piano accompaniment can better cooperate and combine, and the key point of national music is to endow the soul of national music.

\subsection{The important role of piano accompaniment in the practice of ethnic instrumental music}

Music's charm lies in performance and appreciation. In order to give full play to the social significance of ethnic instrumental music, it is necessary to step out of class, go to the stage, and fully demonstrate the performance experience gained by myself through the stage. At this time, higher requirements are put forward for the performer and piano accompaniment art instructor.

The piano accompaniment foreshadows the situation of the work, and the accompaniment process enhances the emotion of the music, which can relieve the psychological pressure of the players, make the performance process more relaxed, confident and enjoyable, and bring the audience a sense of reality of immersive experience. The accompaniment is arranged on the basis of music materials such as tonality, speed and strength of music, which is a supplement of music works. The collaborative interpretation of the works with the players can more fully express the works, and successfully highlight the atmosphere and render the artistic conception, making music richer and more appealing.

\subsection{The important role of piano accompaniment in the creation of ethnic instrumental works}

The piano accompaniment of ethnic instrumental music benefits from the artistic expression of 19th century German and French songs, it was introduced into China in the 20th century and improved step by step. The master professional piano accompaniment art instructor also appeared one after another. Undoubtedly, the performance opportunity at this time also provided a certain display space for the art instructor, showing the important role of accompaniment in the music performance for fans of music.

The piano accompaniment contains a large number of sound patterns and piano playing skills. The basic techniques include intervals, chords, scales, arpeggios, skipping sounds, decorative sounds, etc., for the artistry of national instrumental music works and the assistance in the performance process is not to be underestimated. For example, the accompaniment of the famous slang "Slang" can lay a certain artistic foundation for the playing of the music, provide a certain rhythm, speed, and the arrangement of the bass accompaniment, which can bring out the melody Tone features.

Among the Chinese past five thousand years culture, 56 nationalities have rich and colorful music and a long history. They are the symbol of the spirit and wisdom of all nationalities. If you want to present the musical works of various nationalities to the audience as much as possible, the excellent creation is one side, the creation and cooperation of the accompaniment is another side. It is also very important. Therefore, national instrumentalists and piano accompaniment must constantly improve their literary accomplishments and artistic attainments while understanding the basic music knowledge, and understand the cultural and artistic characteristics of each nation, and the local customs of the Chinese nation. And the traditional virtues to play an important role. This article hopes to make a brief analysis and discussion on the artistic characteristics and playing skills of the piano accompaniment of the national instrumental music works, and to summarize its value, in order to improve the emphasis on piano accompaniment for those who are engaged in the teaching and performance of national musical instruments.

\section{References}

[1] The Past and Present Life of National Instrumental Music and Teaching (Part II)__Comment on the Historical Development of National Instrumental Music and Modern Teaching Art[J]. Zhou Tingting. China Education Journal. 2017(05) 
[2] On the role of Chinese folk instrumental art guidance[J]. Sun Jing. The Voice of the Yellow River. 2017(17)

[3] The role and performance of piano accompaniment in art songs[J]. Zhao Mingchuan. Modern Marketing (College Edition). 2012(01) 